\title{
tst1-positive ST22-MRSA-IVa in healthy Italian preschool children
}

\author{
D. M. Geraci · C. Bonura • M. Giuffrè • \\ A. Aleo - L. Saporito • G. Graziano • \\ R. M. Valenti $\cdot$ C. Mammina
}

Received: 31 October 2013/Accepted: 30 December 2013

(c) Springer-Verlag Berlin Heidelberg 2014

\begin{abstract}
A survey was performed in May 2013 to assess methicillin-resistant Staphylococcus aureus (MRSA) nasal colonization in healthy children attending 26 municipal daycare centres in Palermo, Italy. Of the 500 children, ten (2\%) tested positive. Eight MRSA isolates were tst 1 positive ST22-MRSA-IVa, spa t223; the other two isolates were identified as ST1-IVa and ST398-V, respectively. tst1-positive ST22-MRSA, spa t223 has been previously identified only in the Middle Eastern area.
\end{abstract}

Keywords ST22-MRSA-IVa · Healthy children ·

Daycare centers · Middle Eastern area

\section{Introduction}

Methicillin-resistant Staphylococcus aureus (MRSA) is a major pathogen in healthcare and community settings [1]. One of the most challenging issues in the evolution of MRSA epidemiology is the emergence and spread of community-associated MRSA (CA-MRSA). CA-MRSA strains are generally susceptible to a wider spectrum of non- $\beta$-lactam antibiotics and have smaller staphylococcal cassette chromosome mec (SCCmec) elements (types IV or V) than do healthcare-associated MRSA (HA-MRSA) strains, but they often carry the genes for Panton-Valentine leukocidin. However, blurring of boundaries between the healthcare and the community setting is increasingly

D. M. Geraci · C. Bonura - M. Giuffrè · A. Aleo - L. Saporito · G. Graziano · R. M. Valenti · C. Mammina ( $\square)$ Department of Sciences for Health Promotion and Mother-Child Care "G. D'Alessandro", Università degli Studi di Palermo, Via del Vespro 133, 90127 Palermo, Italy

e-mail: caterina.mammina@unipa.it jeopardizing the differentiation of HA- from CA-MRSA infections.

Only a small number of studies have been conducted to assess MRSA nasal colonization in healthy children in the community [1-4]. Here we report the results of a survey performed to detect MRSA nasal colonization in healthy preschool children in Palermo, southern Italy, an area with high levels of endemic MRSA infections [5].

\section{Methods}

In May 2013 we conducted a point-prevalence study in 26 municipal daycare centres located in Palermo, Italy, hosting children aged $<3$ years. All of the 988 preschool children who attended the centres were considered eligible to be enrolled in the investigation. Informed consent was obtained from the childrens' parents or guardians. A questionnaire was also used to investigate possible risk factors for MRSA carriage, such as household size, number and age of siblings, contact with animals, medical history, symptoms of upper respiratory tract infection at the time of sampling, recent use of antibiotics and prior hospitalization.

Specimens for culture were obtained from both anterior nares with a sterile cotton swab. Each swab was transferred to the microbiology laboratory within $2 \mathrm{~h}$ from sampling, immediately placed in brain-heart infusion broth (OXOID, Basingstoke, UK) and incubated at $35^{\circ} \mathrm{C}$ overnight. Mannitol salt agar plates were then inoculated and, after a further incubation at $35{ }^{\circ} \mathrm{C}$ for $48 \mathrm{~h}$, presumptive $S$. aureus isolates were identified according to standard methods. MRSA isolates were screened for by plating colonies onto oxacillin agar (Mueller-Hinton with oxacillin $6 \mathrm{mg} / \mathrm{L}$ ). Isolates were confirmed as MRSA by the cefoxitin disk diffusion test and PCR assay for the detection of mecA. 
Staphylococcal cassette chromosome mec (SCCmec) typing was performed by the multiplex PCR method described by Milheiriço et al. [6]. Multilocus sequence typing (MLST) was performed by PCR and by the sequencing of seven housekeeping genes, and the MLST allelic profiles and sequence types were assigned by submission to the $S$. aureus MLST database (www.mlst.net). spa typing was also carried out on the MRSA-ST22-IVa isolates using Ridom StaphType software (www.ridom.de/ staphtype/). MRSA clones were defined by both their multilocus sequence type (ST) and their SCCmec type.

Susceptibility to ciprofloxacin, clarithromycin, clindamycin, erythromycin, gentamicin, levofloxacin, linezolid, rifampicin, tetracycline and trimethoprim-sulfamethoxazole was determined using the disk diffusion method. Vancomycin and teicoplanin minimum inhibitory concentrations (MICs) were assessed by the $E$ test. Macrolidelincosamide-streptogramin B $\left(\mathrm{MLS}_{\mathrm{B}}\right)$-inducible phenotypes were detected by the D-zone test as per European Committee on Antimicrobial Susceptibility Testing (EUCAST) guidelines (www.eucast.org/clinical_breakpoints/). Susceptibility testing results were interpreted based on EUCAST clinical breakpoints.

The presence of lukS/lukF-PV and tst 1 genes encoding the PVL and the toxic shock syndrome toxin 1 (TSST-1), respectively, was tested for by PCR [7]. Similarly, ST22-MRSA-IVa isolates were also tested for by PCR for the carriage of enterotoxins $\mathrm{C}$ and $\mathrm{L}$ (sec, sel) using previously described primers and conditions [7].

\section{Results}

Informed consent was obtained from the parents or guardians of 617 children, and nasal swabs were collected from 500 of these. Of these 500 children, 74 (14.8\%) were $S$. aureus carriers. MRSA was detected in ten children ( $13.5 \%$ of S. aureus carriers and $2.0 \%$ of all children) who were attending nine different daycare centres.

Eight MRSA isolates were ST22-MRSA-IVa, spa t223. Antimicrobial susceptibility testing using disk diffusion revealed that five of these eight isolates were susceptible to all non- $\beta$-lactam antibiotics tested and that three isolates were resistant to tetracycline (Table 1). The tst 1 gene was detected in all eight isolates, but the enterotoxin genes sec and sel as well as PVL-encoding genes were not found in any isolate. Of interest, five MSSA isolates from MRSAnegative children were also ST22, spa t223.

Two additional MRSA isolates were identified as ST1IVa and ST398-V, respectively, and proved to be resistant to macrolides and tetracycline. The ST1-IVa isolate showed a $M L S_{B}$-inducible phenotype (Table 1). Both isolates tested negative for $l u k S / l u k F-P V$ and $t s t l$ genes.

The isolates $3 \mathrm{I}$ and $23 \mathrm{U}, \mathrm{ST} 22-\mathrm{IVa}$ and isolate $7 \mathrm{~L}$, ST398-V showed vancomycin MIC values of $2 \mathrm{mg} / \mathrm{L}$, which are at the upper limit of the wild-type MIC distribution.

No association between carriage of the MRSA and specific risk factors arose from the answers of the childrens' parents to the questionnaire. All parents of the MRSA-positive children were of Italian nationality.

Table 1 Pattern of susceptibility to non- $\beta$-lactam antibiotics of ten methicillin-resistant Staphylococcus aureus isolates from healthy preschool children in Palermo, Italy, 2013

\begin{tabular}{|c|c|c|c|c|c|c|c|c|c|c|c|c|c|}
\hline \multirow[t]{2}{*}{ MRSA isolate } & \multirow[t]{2}{*}{ ST-SCCmec } & \multicolumn{12}{|c|}{ non- $\beta$-Lactam antibiotics ${ }^{\mathrm{a}}$} \\
\hline & & CIP & CLR & DA & ERY & $\mathrm{CN}$ & LEV & LZD & RIF & SXT & $\mathrm{TE}$ & VA (MIC) & TEC (MIC) \\
\hline $3 \mathrm{~A}$ & ST1-IVa & S & $\mathrm{R}$ & $S^{b}$ & $\mathrm{R}$ & S & S & S & S & S & $\mathrm{R}$ & $\mathrm{S}(1.5)$ & $\mathrm{S}(0.75)$ \\
\hline $11 \mathrm{~B}$ & ST22-IVa & S & S & S & $\mathrm{S}$ & S & $\mathrm{S}$ & $\mathrm{S}$ & $\mathrm{s}$ & $\mathrm{S}$ & $\mathrm{S}$ & S (1) & S (1) \\
\hline $3 I$ & ST22-IVa & $\mathrm{S}$ & $\mathrm{S}$ & $\mathrm{S}$ & $\mathrm{S}$ & $\mathrm{S}$ & $\mathrm{S}$ & $\mathrm{S}$ & $\mathrm{S}$ & $\mathrm{S}$ & $\mathrm{S}$ & S (2) & $\mathrm{S}(0.5)$ \\
\hline $2 \mathrm{~L}$ & ST22-IVa & S & $S$ & $\mathrm{~S}$ & $\mathrm{~S}$ & $S$ & $S$ & $\mathrm{~S}$ & $S$ & $\mathrm{~S}$ & $\mathrm{R}$ & S (1) & S $(0.38)$ \\
\hline $7 \mathrm{~L}$ & ST398-V & $\mathrm{S}$ & $\mathrm{R}$ & $\mathrm{R}$ & $\mathrm{R}$ & $\mathrm{S}$ & $\mathrm{S}$ & $\mathrm{S}$ & $\mathrm{S}$ & $\mathrm{S}$ & $\mathrm{R}$ & S (2) & S $(0.75)$ \\
\hline $7 \mathrm{M}$ & ST22-IVa & $\mathrm{S}$ & $\mathrm{S}$ & $\mathrm{S}$ & $\mathrm{S}$ & $\mathrm{S}$ & $\mathrm{S}$ & $\mathrm{S}$ & $\mathrm{S}$ & $\mathrm{S}$ & $\mathrm{S}$ & $\mathrm{S}(1.5)$ & $S(0.75)$ \\
\hline $7 \mathrm{~S}$ & ST22-IVa & S & $S$ & S & $S$ & $S$ & S & S & S & S & $\mathrm{S}$ & $\mathrm{S}(1.5)$ & $\mathrm{S}(0.5)$ \\
\hline $23 \mathrm{U}$ & ST22-IVa & S & $S$ & $\mathrm{~S}$ & $\mathrm{~S}$ & $S$ & $S$ & $\mathrm{~S}$ & $S$ & $\mathrm{~S}$ & $\mathrm{~S}$ & S (2) & $\mathrm{S}(0.5)$ \\
\hline $16 \mathrm{~V}$ & ST22-IVa & S & $S$ & $\mathrm{~S}$ & $\mathrm{~S}$ & $S$ & $S$ & $\mathrm{~S}$ & $S$ & $\mathrm{~S}$ & $\mathrm{R}$ & $\mathrm{S}(1.5)$ & $\mathrm{S}(0.5)$ \\
\hline $17 \mathrm{X}$ & ST22-IVa & $\mathrm{S}$ & $\mathrm{S}$ & $\mathrm{S}$ & $\mathrm{S}$ & $\mathrm{S}$ & $\mathrm{S}$ & $\mathrm{S}$ & $\mathrm{S}$ & $\mathrm{S}$ & $\mathrm{R}$ & $\mathrm{S}(1.5)$ & S $(0.38)$ \\
\hline
\end{tabular}

MRSA, Methicillin-resistant Staphylococcus aureus; S, susceptible; R, resistant; MIC, minimum inhibitory concentration

${ }^{a}$ CIP, Ciprofloxacin $(5 \mu \mathrm{g})$; CLR, clarithromycin (15 $\left.\mu \mathrm{g}\right)$; DA, clindamycin $(2 \mu \mathrm{g})$; ERY, erythromycin $(5 \mu \mathrm{g})$; CN, gentamicin (10 $\left.\mu \mathrm{g}\right)$; LEV, levofloxacin $(5 \mu \mathrm{g})$; LZD, linezolid (30 $\mu \mathrm{g})$; RIF, rifampicin $(5 \mu \mathrm{g})$; SXT, sulfamethoxazole-trimethoprim $(25 \mu \mathrm{g})$; TE, tetracycline $(30 \mu \mathrm{g})$; VA, vancomycin; TEC, teicoplanin

b Macrolide-lincosamide-streptogramin B $\left(\mathrm{MLS}_{\mathrm{B}}\right)$-inducible phenotype 
According to the information provided by the questionnaires, none of the children had been hospitalized or had taken antibiotics in the 3 months prior to their nasal sampling. Moreover, none of the family members of the child carrying the ST398-MRSA-V isolate reported any direct or indirect exposure to livestock.

\section{Discussion}

Little data are available on the prevalence and strains of MRSA carriage in healthy children in the community [14]. To the best of our knowledge, no data are available from Italy, a European country which is categorized as being highly endemic for MRSA infection [5]. Our survey detected a $2.0 \%$ prevalence of MRSA carriage in healthy children attending 26 municipal daycare centres in Palermo. A similar prevalence $(2.3 \%$; $95 \%$ confidence interval 1.8-2.7) was reported by a recent meta-analysis of children recruited in kindergartens and schools or during well-child visits in various countries around the world [1]. However, in our setting an unexpected finding was the predominance of the tst1-positive ST22-MRSA, spa t223 strain. This strain, previously identified as a Middle Eastern variant of UK-EMRSA-15, appears to be identical to the "Gaza strain" described by Biber et al. [8] as the predominant MRSA clone in healthy children and their parents living in the Gaza strip [8,9]. MRSA strains with similar characteristics have also been reported from Saudi Arabia, Abu Dhabi and Egypt [10]. A spa t223 strain has also been recently described as the second most common MRSA strain among 227 healthy volunteers from a Jordanian community [11]. The possible relationships between this MRSA strain and the ST22-IVa isolates which have been identified in several healthcare settings in our geographic area deserves further investigation [12]. The most likely origin of the "Gaza strain", according to the hypothesis of Biber et al. [9], should be a locally prevalent ST22-MSSA spa t223 strain. However, our findings suggest that this strain has spread into a wider area, including the Mediterranean region. The detection in our setting of ST22-MSSA, spa t223 appears to be consistent with the hypothesis of a local evolution towards resistance to methicillin, which has been suggested as an alternative to the import of EMRSA-15 into the Middle Eastern area [9]. Further intensive molecular investigations on these strains are needed to reliably track their evolution and dissemination routes.

An alarming finding is our detection of MRSA isolates with vancomycin MIC values of $2 \mathrm{mg} / \mathrm{L}$ in healthy community subjects. Indeed, despite these strains being categorized by EUCAST guidelines as susceptible, several clinical studies report high rates of unfavourable clinical response in patients infected with MRSA isolates having MICs on the borderline of the wild-type MIC distribution [13].

Our study has several limitations. First, it is a crosssectional study with a relatively small sample size. Moreover, because access to municipal child daycare centres is primarily directed towards lower income families, our results may not be generalizable to the city population as a whole. Secondly, our MRSA screening method using mannitol salt agar may have been less specific and sensitive than isolation on chromogenic agar media, even though we used a broth enrichment step to enhance MRSA detection [14].

In conclusion, the molecular epidemiological characteristics of MRSA isolates from healthy children in Palermo, Italy, appear to be similar to those reported in some Middle Eastern countries. Further studies focusing on the molecular characterization of carriage and clinical isolates of CA- and HA-MRSA are needed to provide more informative data on the burden and evolution of MRSA in our geographic area.

Acknowledgments We are indebted to the staff of the municipal daycare centers of Palermo, Italy, who made it possible to perform this investigation. This study was supported by institutional funding.

\section{Conflict of interest None.}

Ethical standards The study was approved by the ethical committee of the Azienda Ospedaliero-Universitaria Policlinico "Paolo Giaccone", Palermo, Italy.

\section{References}

1. Gesualdo F, Bongiorno D, Rizzo C, Bella A, Menichella D, Stefani S, Tozzi AE. MRSA nasal colonization in children: prevalence meta-analysis, review of risk factors and molecular genetics. Pediatr Infect Dis J. 2013;32:479-85.

2. Lamaro-Cardoso J, de Lencastre H, Kipnis A, Pimenta FC, Oliveira LS, Oliveira RM, Nouer SS, Aires-de-Sousa M, Milheiriço C, Andrade AL. Molecular epidemiology and risk factors for nasal carriage of Staphylococcus aureus and methicillin-resistant S. aureus in infants attending day care centers in Brazil. J Clin Microbiol. 2009;47:3991-7.

3. Lee J, Sung JY, Kim YM, Oh CE, Kim HB, Choi EH, Lee HJ. Molecular characterization of methicillin-resistant Staphylococcus aureus obtained from the anterior nares of healthy Korean children attending daycare centers. Int J Infect Dis. 2011;15:e558-63.

4. Ho PL, Chiu SS, Chan MY, Gan Y, Chow KH, Lai EL, Lau YL. Molecular epidemiology and nasal carriage of Staphylococcus aureus and methicillin-resistant $S$. aureus among young children attending day care centers and kindergartens in Hong Kong. J Infect. 2012;64:500-6.

5. European Centre for Disease Control and Prevention (ECDC). Antimicrobial resistance surveillance in Europe. Annual report of the European Antimicrobial Resistance Surveillance Network (EARS-Net), 2012. Stockholm: ECDC; 2013.

6. Milheiriço C, Oliveira DC, de Lencastre H. Update to the multiplex PCR strategy for assignment of mec element types in 
Staphylococcus aureus. Antimicrob Agents Chemother. 2007;51: 3374-7.

7. Karauzum H, Ferry T, de Bentzmann S, Lina G, Bes M, Vandenesch F, Schmaler M, Berger-Bächi B, Etienne J, Landmann R. Comparison of adhesion and virulence of two predominant hospital-acquired methicillin-resistant Staphylococcus aureus clones and clonal methicillin-susceptible $S$. aureus isolates. Infect Immun. 2008;76:5133-8.

8. Biber A, Abuelaish I, Rahav G, Raz M, Cohen L, Valinsky L, Taran D, Goral A, Elhamdany A, Regev-Yochay G. PICR Study Group. A typical hospital-acquired methicillin-resistant Staphylococcus aureus clone is widespread in the community in the Gaza strip. PLoS One. 2012;7:e42864.

9. Monecke S, Coombs G, Shore AC, Coleman DC, Akpaka P, Borg M, Chow H, Ip M, Jatzwauk L, Jonas D, Kadlec K, Kearns A, Laurent F, O'Brien FG, Pearson J, Ruppelt A, Schwarz S, Scicluna E, Slickers P, Tan HL, Weber S, Ehricht R. A field guide to pandemic, epidemic and sporadic clones of methicillin-resistant Staphylococcus aureus. PLoS One. 2011;6:e17936.

10. Monecke S, Skakni L, Hasan R, Ruppelt A, Ghazal SS, Hakawi A, Slickers P, Ehricht R. Characterisation of MRSA strains isolated from patients in a hospital in Riyadh, Kingdom of Saudi Arabia. BMC Microbiol. 2012;12:146.
11. Al-Bakri AG, Al-Hadithi H, Kasabri V, Othman G, Kriegeskorte A, Becker K. The epidemiology and molecular characterization of methicillin-resistant staphylococci sampled from a healthy Jordanian population. Epidemiol Infect. 2013;141:2384-91.

12. Mammina $\mathrm{C}$, Calà $\mathrm{C}$, Bonura $\mathrm{C}, \mathrm{Di}$ Carlo $\mathrm{P}$, Aleo $\mathrm{A}$, Fasciana $\mathrm{T}$. Giammanco A; EPI-MRSA Working Group. Polyclonal non multiresistant methicillin resistant Staphylococcus aureus isolates from clinical cases of infection occurring in Palermo, Italy, during a one-year surveillance period. Ann Clin Microbiol Antimicrob. 2012;19(11):17.

13. Uçkay I, Bernard L, Buzzi M, Harbarth S, François P, Huggler E, Ferry T, Schrenzel J, Renzoni A, Vaudaux P, Lew DP. High prevalence of isolates with reduced glycopeptide susceptibility in persistent or recurrent bloodstream infections due to methicillinresistant Staphylococcus aureus. Antimicrob Agents Chemother. 2012;56:1258-64.

14. Clinical and Laboratory Standards Institute (CLSI). Surveillance for methicillin resistant Staphylococcus aureus: principles, practices, and challenges; a report. CLSI document X07-R. Waine: CLSI; 2010. 